\title{
RESTORATION OF ACTUAL TRACK GEOMETRY USING DATA MEASURED BY AN INERTIAL TROLLEY
}

\author{
YASUKUNI NAGANUMA \& TAKAYUKI UEMATSU \\ Central Japan Railway Company, Komaki, Japan
}

\begin{abstract}
This paper describes a method for restoring actual track geometry using data measured with a newly developed inertial trolley and the application of this method. First, we present the result of an estimation of actual geometry obtained through the application of an inverse filter, which is a classical digital signal processing technique. Recently, the authors developed a method of restoring actual track geometry using a Bayesian filter. In this paper, we show that the restoration by the Kalman filter, which is the most popular Bayesian filter, is superior to the inverse filter. In addition, as an application of this technique, we proposed a compensation method for gauge and cant irregularity data measured by means of a three-wheel trolley, and we demonstrated that gauge irregularity as defined may be obtained without any additional sensors.
\end{abstract}

Keywords: actual track geometry, track geometry measuring trolley, inverse filter, Kalman filter.

\section{INTRODUCTION}

The main method for measuring railway track is to use a track geometry car and either the versine method [1] or inertial method [2]. Although the versine data and D1, D2 and D3 track irregularity prescribed in EN 13848 are different from the actual track geometry, they provide sufficient information to maintain good ride comfort and running safety. However, in order to properly manage track shape and further improve riding comfort, it is necessary to back-calculate actual track geometry from track irregularity data. Also, actual track geometry is indispensable as input data for dynamic simulation of the vehicle. Therefore, the authors developed a method that uses a Bayesian estimation filter for actual track geometry reconstruction [3], [4]. In addition, LR-S100, a track geometry measuring trolley using the inertia method, was recently developed [5]. Because this trolley is much more accurate than conventional products, there is a possibility that the measured data can be used to restore the actual track geometry.

Within the aforementioned backdrop, the first objective of this paper is to apply the conventional track geometry restoration method, which has been applied to track geometry car data, and the newly developed Bayesian estimation method to measurement data obtained with the newly developed inertial trolley. Also, sometimes cost, space or other restrictions do not allow the sensor to be mounted in the proper position on a track geometry measuring trolley, which is required to be small and lightweight, and measurement results are approximate values. For example, with a three-wheel inertial trolley, gauge and cant are unable to be measured at the same corresponding points on the left and right rails [6]. The second objective of this paper is to propose a method to compensate for gauge and cant measured by the three-wheel inertial trolley by using restored actual track geometry.

\section{INERTIAL TROLLEY (LR-S100)}

Fig. 1 shows a track geometry measuring trolley that uses an inertial sensor called LR-S100, which was successfully developed in 2015. This trolley employs a newly developed track measuring method named as the differential-difference method (DD-method). Fig. 2 shows the measuring principle of the DD-method. The difference in the local gradient $(r(\xi))$ is 


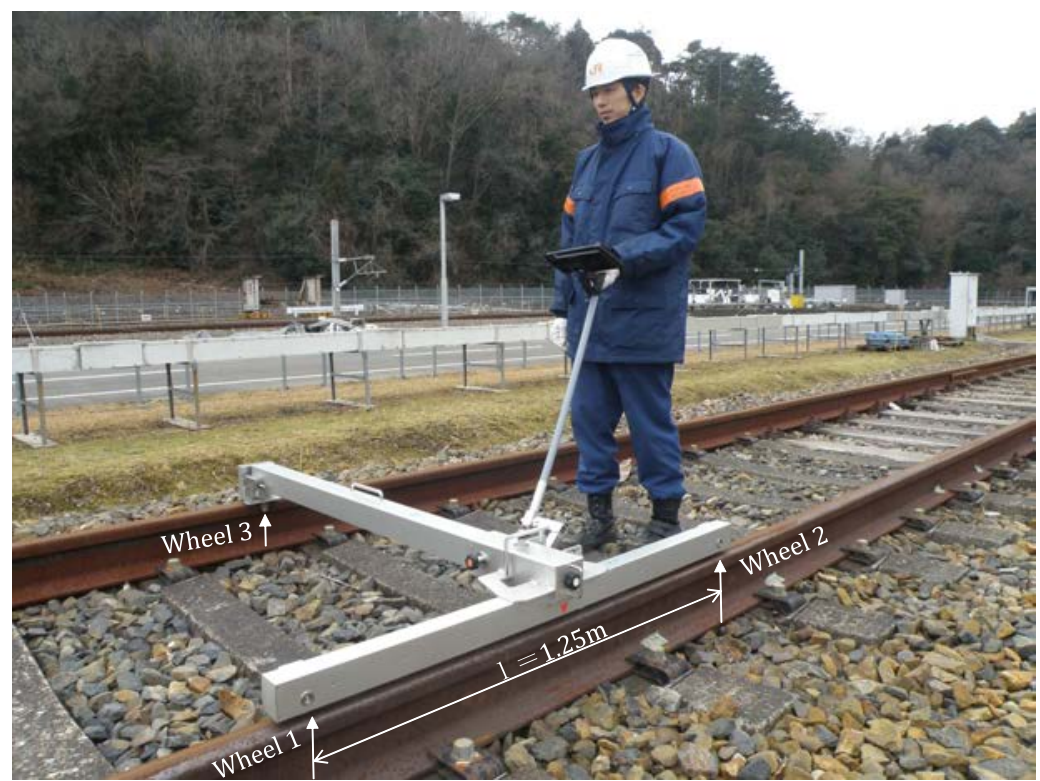

Figure 1: Inertial track geometry measuring trolley (LR-S100).

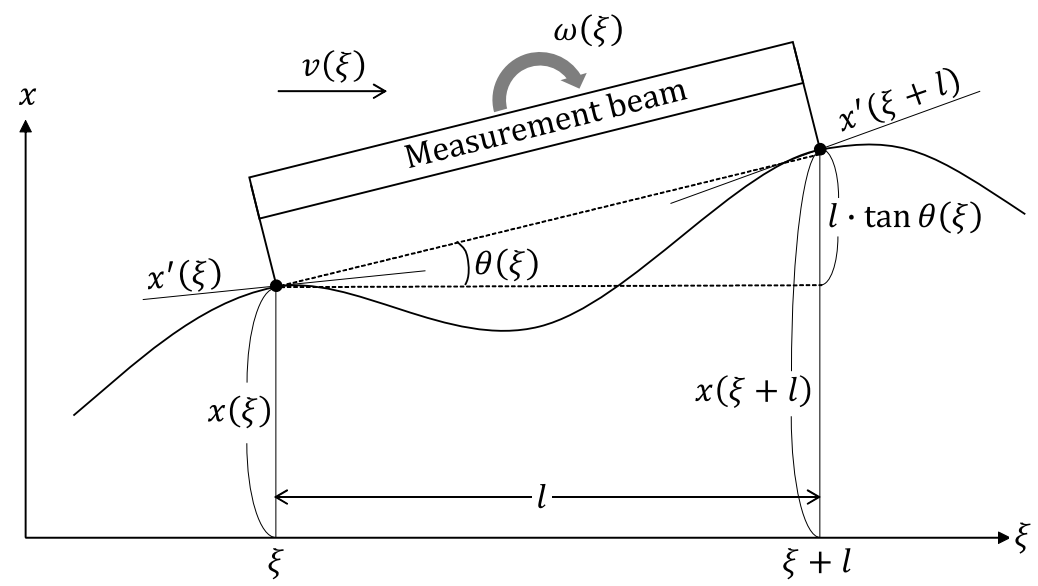

Figure 2: Differential difference method (DD-method).

obtained by eqn (1) using the angular velocity $(\omega(\xi))$ measured by the gyroscope.

$$
\begin{aligned}
r(\xi) & =\frac{d}{d \xi}(x(\xi+l)-x(\xi))=\frac{d}{d \xi}(l \cdot \tan \theta(\xi)) \\
& =l \frac{1}{\cos ^{2} \theta(\xi)} \cdot \frac{d}{d \xi} \theta(\xi)=l \underbrace{\frac{1}{\cos ^{2} \theta(\xi)}}_{\cong 1.0} \cdot \underbrace{\frac{d}{d t} \theta(\xi)}_{\omega(\xi)} \cdot \underbrace{\frac{d t}{d \xi} \cong \frac{\omega(\xi)}{v(\xi)}}_{1 / v(\xi)}
\end{aligned}
$$




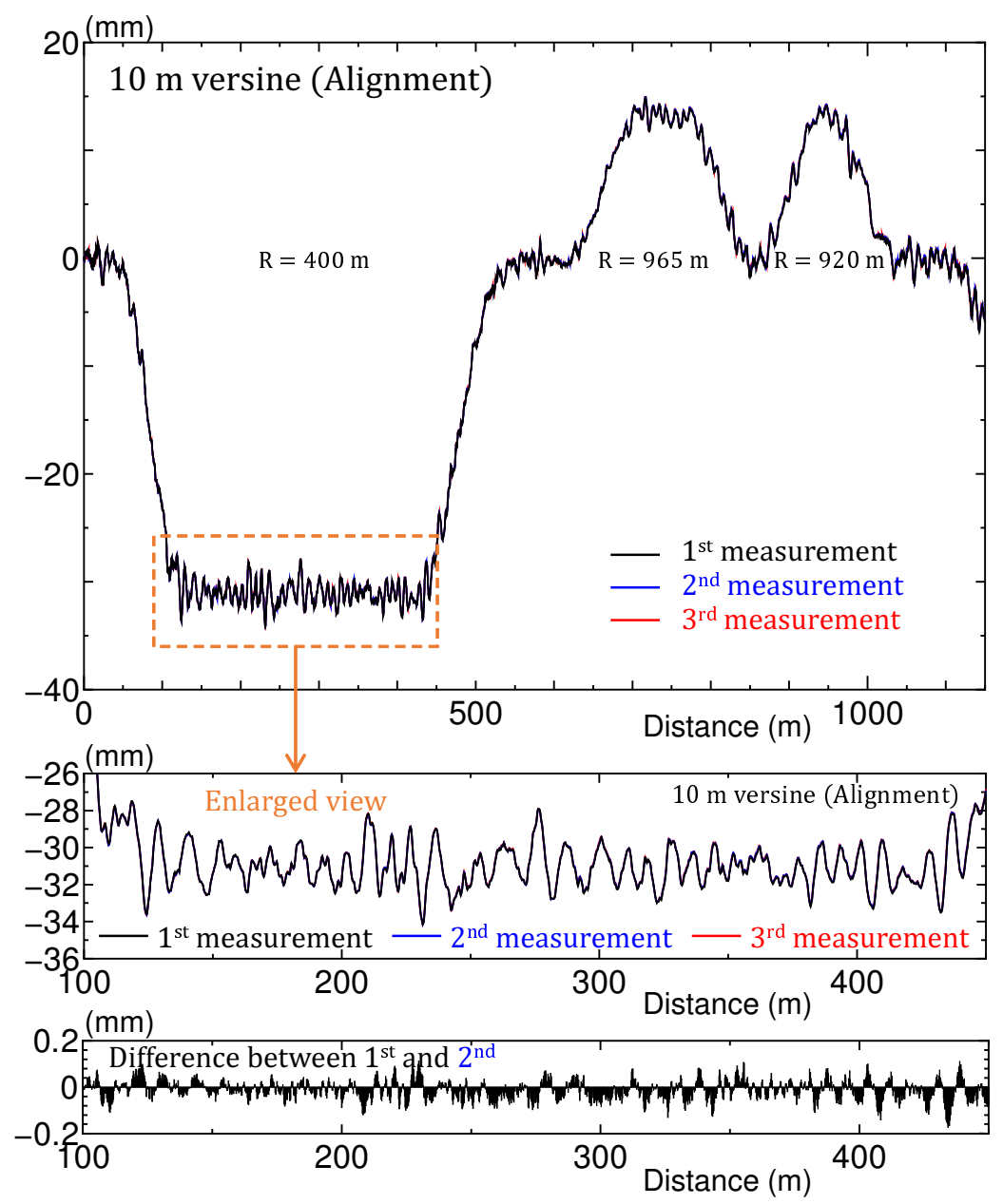

Figure 3: Repeatability of $10 \mathrm{~m}$ versine alignment measured by LR-S100.

where, $l$ is the length of the trolley measuring beam, $v(\xi)$ is the measuring velocity and an approximation of $\cos \theta(\xi) \cong 1$ is introduced because $\theta(\xi)$ is relatively small. This DDsignal data may be completely converted to $2.5 \mathrm{~m}$ versine by the simple and stable calculus of moving summation [5].

Fig. 3 shows the repeatability of the $10 \mathrm{~m}$ versine alignment calculated from the LRS100 measurement results. The LR-S100 is a simple structure that is compact, lightweight $(13.4 \mathrm{~kg})$ and does not have a complex mechanism, but it has high repeatability accuracy that surpasses that of conventional track measuring trolleys.

As shown in the lower graph of Fig. 3, the difference between the 1st measurement and 2nd measurement is within $\pm 0.2 \mathrm{~mm}$. 

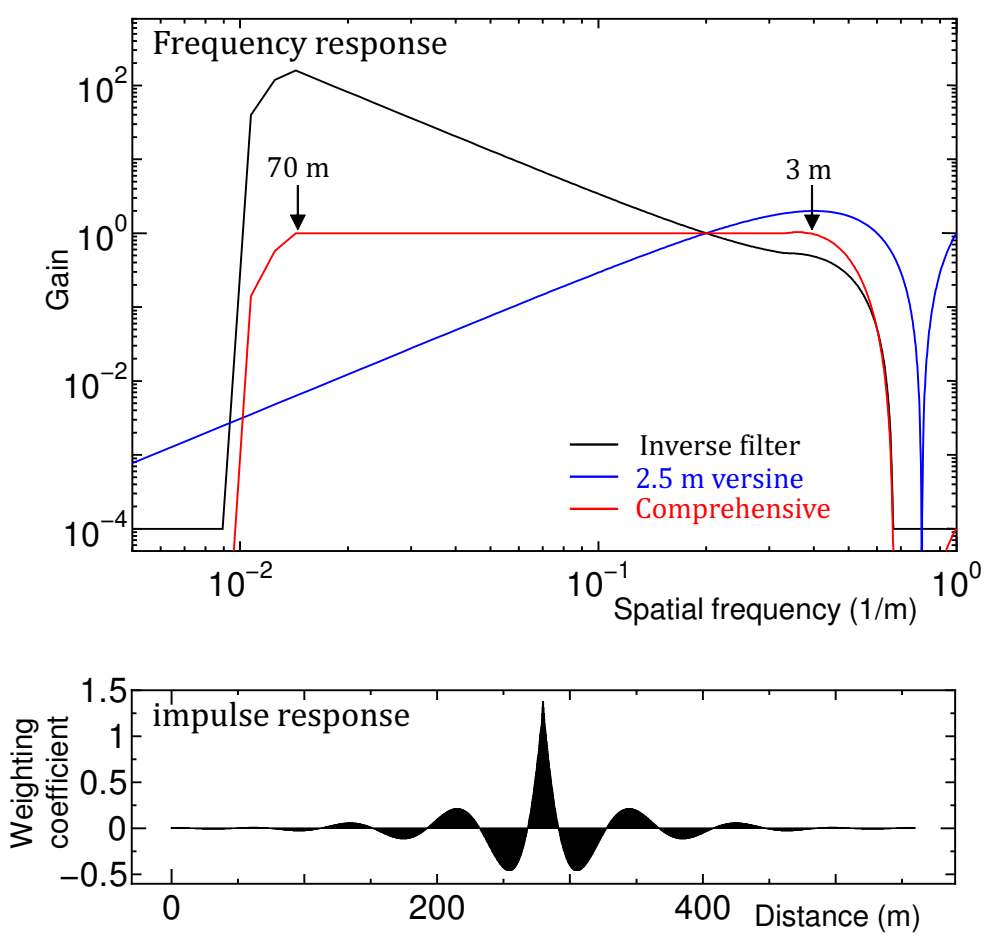

Figure 4: Inverse filter.

\section{RESTORATION OF ACTUAL TRACK GEOMETRY}

\subsection{Inverse filtering}

In Japan, the inverse filtering is mainly used to restore actual track geometry [7], [8]. This method aims to continuously reconstruct actual track geometry $x(n)$ from measured track irregularity $y(n)$ using the following eqn (2):

$$
x(n)=\sum_{k=0}^{N-1} b(k) \cdot y(n-k) .
$$

FIR filter of order $N$ with the impulse response $b(k)$ is obtained by inverse Fourier transformation of the inverse characteristic of the measuring system. Fig. 4 shows the frequency response function and impulse response function for an inverse filter for $2.5 \mathrm{~m}$ versine measurement by LR-S100 inertial trolley. Unfortunately, a complete restoration of the actual track geometry is not possible because the gain of the filter approaches infinity as the spatial frequency lowers. Therefore, a restricted restoration of actual track geometry with 3-70 $\mathrm{m}$ wavelength bands is used in this study. This is equivalent to a combination of D1 and D2 track irregularity as specified in EN-13848. Fig. 5. shows three restorations calculated using the inverse filter. In the case of an FIR filter, the output signal has a transient response, which decreases calculation precision, in the beginning and end, and it is $1 / 2$ length of the 


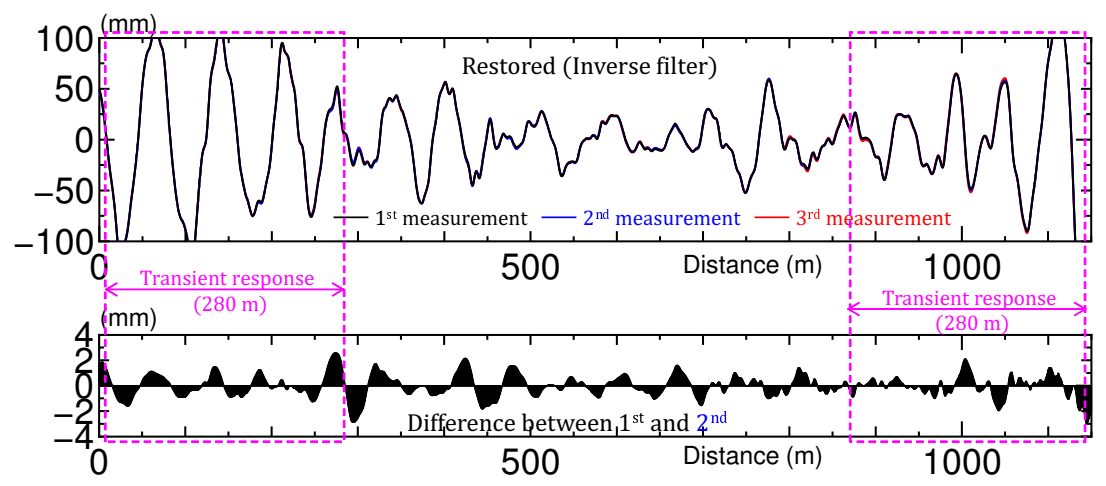

Figure 5: Repeatability of alignment geometry restored by inverse filter.

impulse response. Therefore, the transient response length of the designed inverse filter is $280 \mathrm{~m}$. The range of the transient responses are shown in Fig. 5. Since the repeatability error of the restored track geometry in the wavelength $3-70 \mathrm{~m}$ band is within $3 \mathrm{~mm}$, it may be used as control data for the tamping machine. Along with the original measurements, Fig. 6 shows the $10 \mathrm{~m}$ versine alignment recalculated from the restored track geometry. Since the inverse filter uses the restricted wavelength as described above, the wavelength component outside the restoration band appears as the difference in Fig. 6.

\subsection{Kalman filtering}

In previous studies, we developed a new restoration technique that applies a Bayesian estimation filter to solve the inverse problem of restoring actual track geometry from track irregularity data [3], [4]. When the Kalman filter, one of the most popular Bayesian estimators, is applied, the track irregularity measurements are expressed by linear Gaussian state space models in eqn (3) and (4):

$$
\begin{array}{ll}
\boldsymbol{x}_{n}=F \boldsymbol{x}_{n-1}+G \boldsymbol{v}_{n} & \text { (system model), } \\
\boldsymbol{y}_{n}=H \boldsymbol{x}_{n}+\boldsymbol{w}_{n} & \text { (observation model) } .
\end{array}
$$

Here, process noise $\boldsymbol{v}_{n}$ is assumed to be drawn from a zero mean multivariate normal distribution with covariance $Q_{n}$, and observation noise $\boldsymbol{w}_{n}$ to be a zero mean Gaussian white noise with covariance $R_{n}$. A random walk model or a trend model is used for the system equation (eqn (3)) to apply the Bayesian estimation filter to inverse analysis. The secondorder trend model $\left(x_{n}=2 x_{n-1}-x_{n-2}+v_{n}\right)$ was used in this study. State vector $\boldsymbol{x}_{n}$ which is the track geometry, observation vector $\boldsymbol{y}_{n}$ which is the track geometry measurement data, and matrices $F$ and $G$ are as follows:

$$
\boldsymbol{x}_{n}=\left(x_{n}, x_{n-1}, \ldots, x_{n-k+1}\right)^{T}, \quad \boldsymbol{y}_{n}=\left(y_{n}, y_{n-1}, \ldots, y_{n-k+1}\right)^{T}
$$




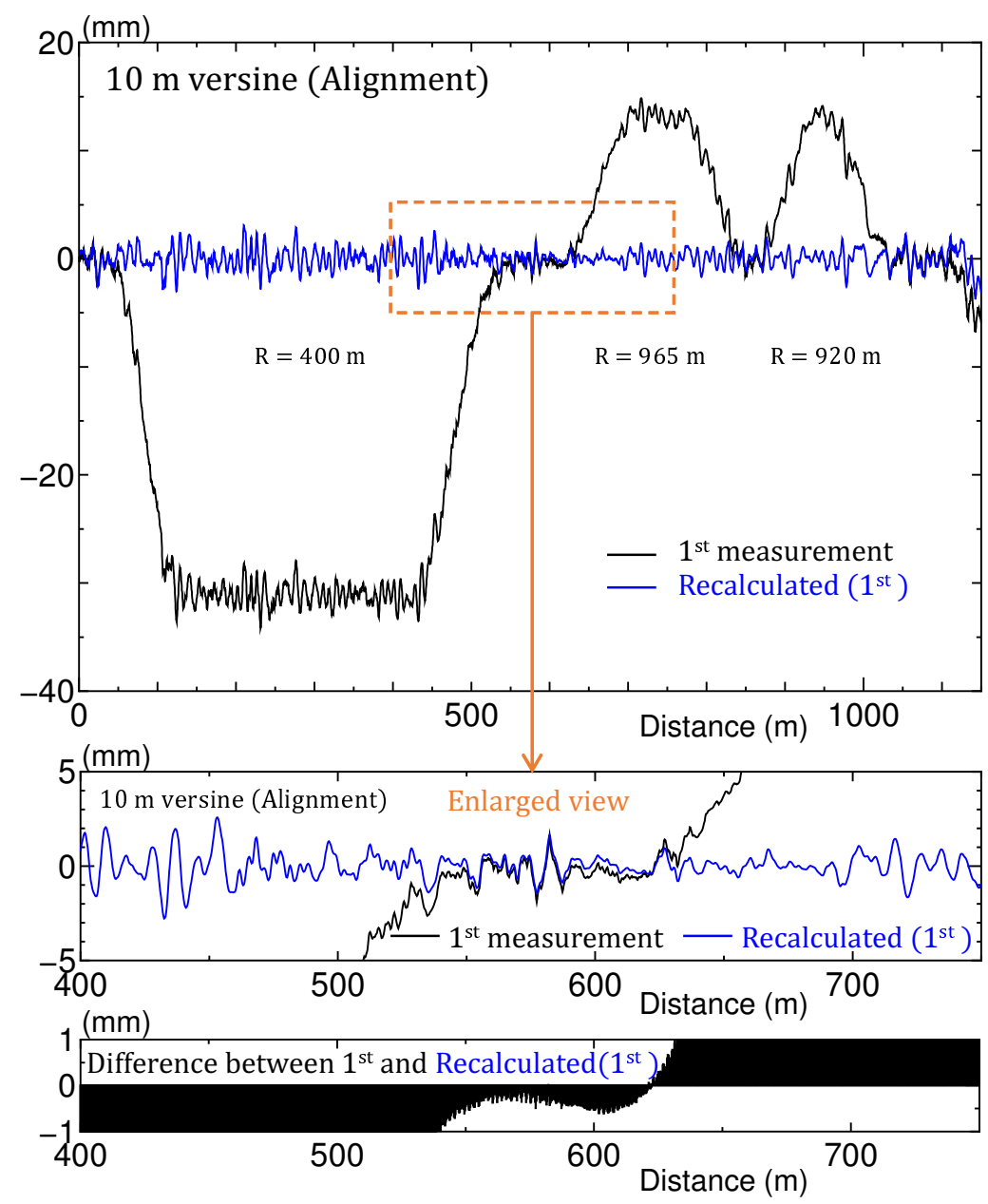

Figure 6: Recalculation results of $10 \mathrm{~m}$ versine alignment.

$$
F=\left(\begin{array}{ccccc}
2 & -1 & 0 & \cdots & 0 \\
1 & 0 & 0 & \cdots & 0 \\
0 & 1 & 0 & \cdots & 0 \\
\vdots & \ddots & \ddots & \ddots & \vdots \\
0 & \cdots & 0 & 1 & 0
\end{array}\right), \quad G=\left(\begin{array}{c}
1 \\
0 \\
0 \\
\vdots \\
0
\end{array}\right)
$$

When the data sampling interval is considered to be $0.25 \mathrm{~m}$, transfer function of the $2.5 \mathrm{~m}$ versine measurement yields eqn (7), so the observation matrix $H$ in eqn (4) is eqn (8).

$$
\begin{gathered}
H_{V S}(z)=-\frac{1}{2}+z^{-5}-\frac{1}{2} z^{-10} \\
H=\left(\begin{array}{lllllllllll}
-0.5 & 0 & 0 & 0 & 0 & 1 & 0 & 0 & 0 & 0 & -0.5
\end{array}\right) .
\end{gathered}
$$


Inverse analysis of the track geometry using the Kalman filter is enabled based on eqns (9) to (13). In this step of the calculation, the track geometry $\boldsymbol{x}_{n}$ is reconstructed as the state variable vector sequentially and stably as follows:

$$
\begin{aligned}
\text { (prediction) } & \\
\boldsymbol{x}_{n \mid n-1} & =F \boldsymbol{x}_{n-1 \mid n-1}, \\
V_{n \mid n-1} & =F V_{n-1 \mid n-1} F^{T}+G Q G^{T}, \\
\text { (filtering) } & \\
K_{n} & =V_{n \mid n-1} H^{T}\left(H V_{n \mid n-1} H^{T}+R\right)^{-1}, \\
\boldsymbol{x}_{n \mid n} & =\boldsymbol{x}_{n \mid n-1}+K_{n}\left(\boldsymbol{y}_{n}-H \boldsymbol{x}_{n \mid n-1}\right), \\
V_{n \mid n} & =\left(I-K_{n} H\right) V_{n \mid n-1} .
\end{aligned}
$$

Fig. 7 shows the actual track geometry estimated from the $2.5 \mathrm{~m}$ versine alignment using the Kalman filter. The parameters used in the Kalman filter were $\sigma_{v}^{2}=0.02$ and $\sigma_{w}^{2}=1.0 \times 10^{-6}$.

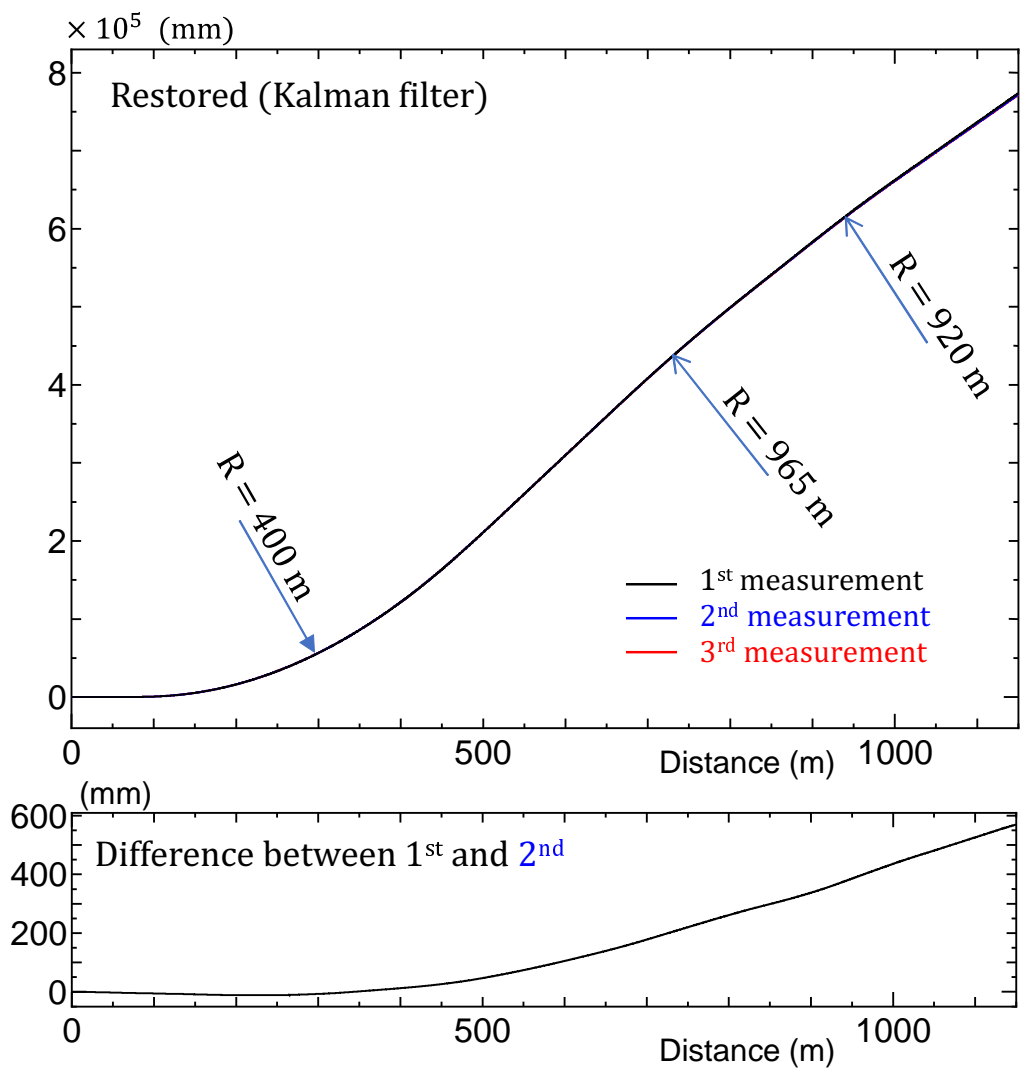

Figure 7: Repeatability of alignment restoration results (Kalman filter). 


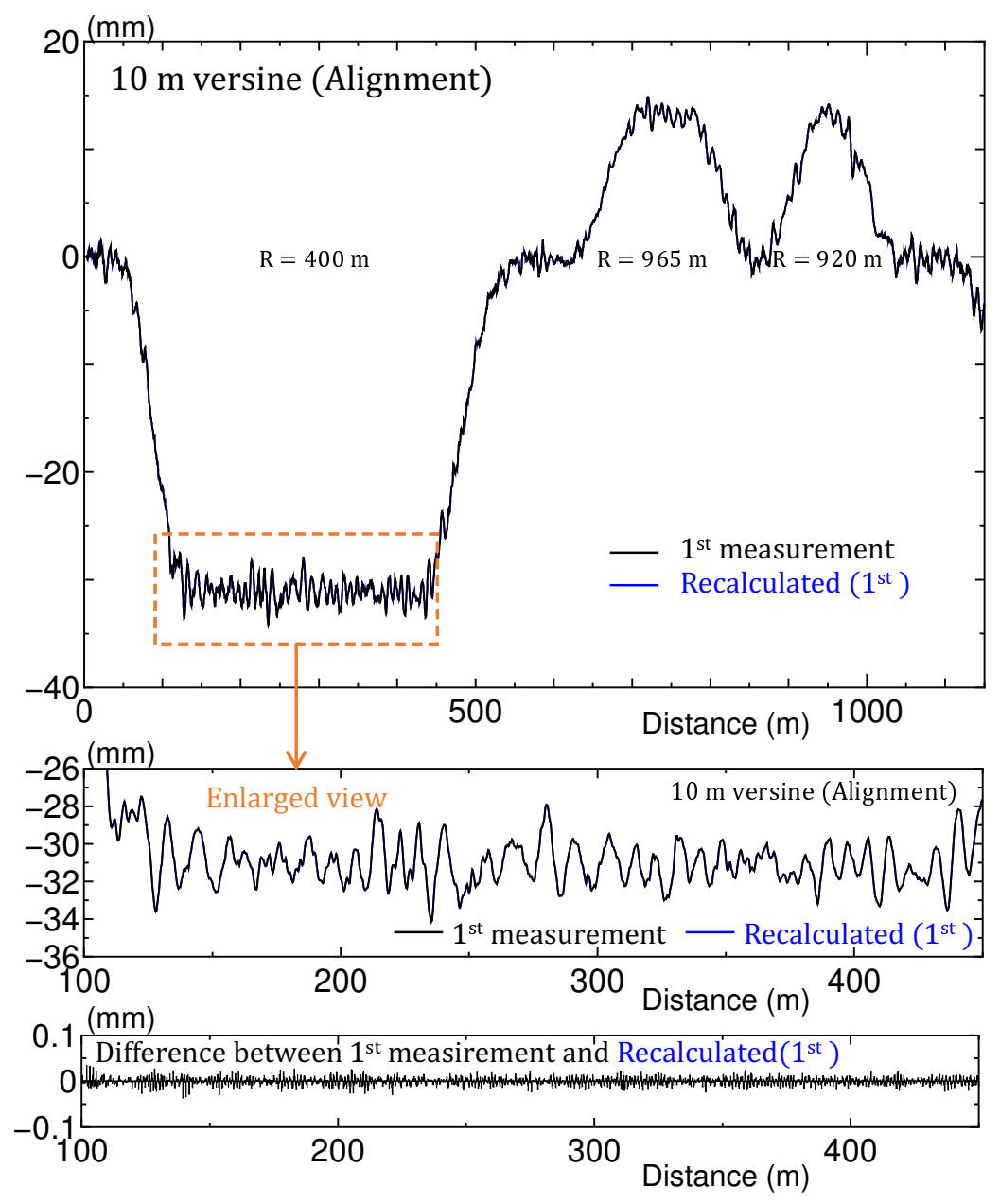

Figure 8: Recalculation results of $10 \mathrm{~m}$ versine alignment (Kalman filter).

Looking at the calculation results, the long wavelength components including the track geometry are restored, it is useful information for maintaining the track shape and improving further riding comfort. In restoring the distance of $1200 \mathrm{~m}$, the difference between the two results is about $60 \mathrm{~cm}$, and it can be said that it is highly accurate as a method without reference point information. For further utilization in future such as construction of new lines, it is better to add information on several reference points. Fig. 8 shows the $10 \mathrm{~m}$ versine alignment recalculated from the restored track geometry. Noteworthy is that recalculated $10 \mathrm{~m}$ versine, even in the curved sections, very well matches the measured value. This proves that the restoration result by the Kalman filter completely retains the information of the measurement data including long wavelength components. This advantage of the Kalman filter restoration enables various applications in track technology. 


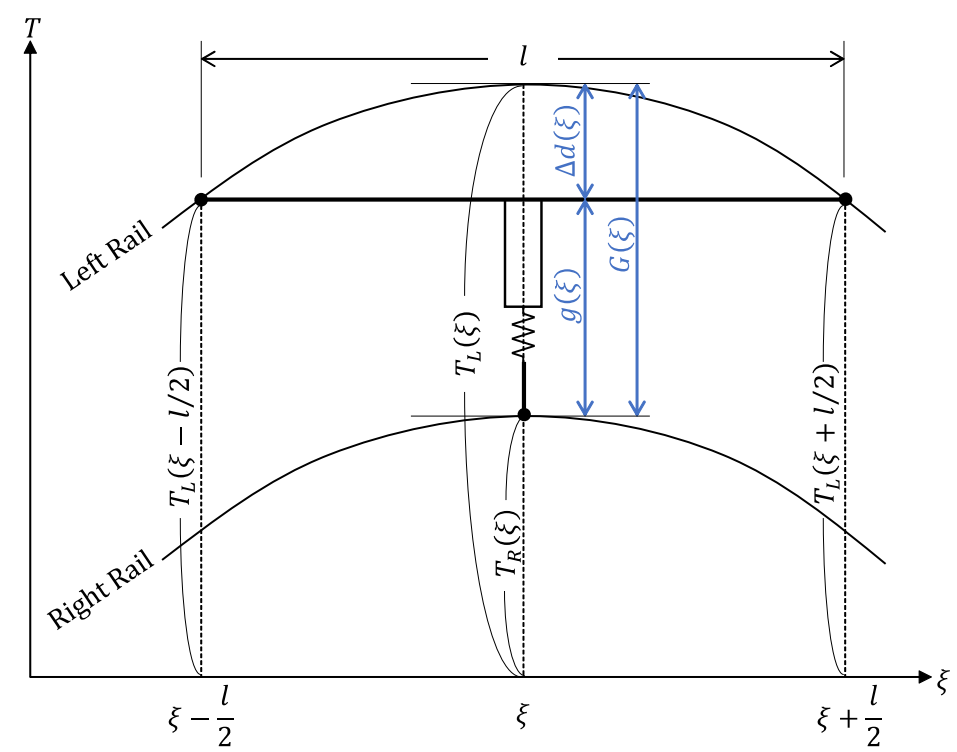

Figure 9: Gauge irregularity measured by three-wheel inertial trolley.

\section{APPLICATION OF ACTUAL TRACK GEOMETRY RECONSTRUCTION \\ TECHNIQUE (LR-S100 GAUGE AND CANT MEASUREMENT COMPENSATION)}

In this section, an example is given of application of the actual track geometry restoration technique described above. This is a method of compensating for gauge and cant measured by a three-wheel inertial trolley, such as LR-S100. Since the LR-S100 has three wheels, the cant and level are approximate measurements. Fig. 9 shows the gauge measurement by the inertial trolley of the three wheel structure. In Fig. 9, the gauge irregularity $g(\xi)$ measured by the three-wheel inertial trolley is expressed in eqn (14):

$$
g(\xi)=\frac{T_{L}\left(\xi-\frac{l}{2}\right)+T_{L}\left(\xi+\frac{l}{2}\right)}{2}-T_{R}(\xi) .
$$

Here, $T_{L}$ is the actual track geometry of the left rail, $T_{R}$ is that of the right rail, and $l$ is the length of the trolley measuring beam. And, legal gauge $(G)$ is defined in eqn (15):

$$
G(\xi)=T_{L}(\xi)-T_{R}(\xi)
$$

Therefore, as shown in eqn (16), the difference $\Delta d$ becomes versine where the length between two wheels is chord length $l$, which is equal to $1.25 \mathrm{~m}$ chord versine in LR-S100:

$$
\begin{aligned}
\Delta d(\xi) & =G(\xi)-g(\xi) \\
& =T_{L}(\xi)-\frac{T_{L}\left(\xi-\frac{l}{2}\right)+T_{L}\left(\xi+\frac{l}{2}\right)}{2} .
\end{aligned}
$$

This $\Delta d$ is very small when the straight section or the curve radius $\left(R_{c}\right)$ is large, but this difference term is too large to ignore in the case of a subway, tramway or other railway where the curve radius is small. Fig. 10 is a comparison between the three points gauge $(\mathrm{g})$ 
of LR-S100 and the correct two points gauge $(G)$ measured by the conventional trolley in the tramway track, and the minimum curve radius of this section is $23 \mathrm{~m}$. The difference may be calculated by the classic formula $\left(l^{2} / 8 R_{c}\right)$ and it will also be approximately $8.5 \mathrm{~mm}$. In LR-S100, $\Delta d$ is estimated by a simple method using $2.5 \mathrm{~m}$ versine alignment, and gauge is corrected in real time. In this chapter, a more accurate compensation method that uses the actual track geometry described above was examined.

For example, when the actual track geometry $T_{L}$ of the left rail is obtained using the method described in Chapter 3, first the eqn (14) is used to calculate track geometry $T_{R}$ of the opposite right rail. Next, using eqn (15), we can obtain a correct gauge as defined. Fig. 11 shows a comparison of the result compensating three-point gauge using the actual track geometry estimated by Kalman filter and the two-point gauge measured with a conventional trolley. As is apparent from the figure, gauge irregularity measured with LR-S100, which is a three-wheel structure, is compensated, and the correct gauge is obtained. Fig. 12 shows the repeatability of the compensated gauge. Except for the turnout section, the difference between the two measurements is within $0.3 \mathrm{~mm}$, and it is confirmed that the repeatability after the compensation is sufficiently high.

Cant irregularity measured by a three-wheel trolley may be compensated in exactly the same manner as gauge irregularity.

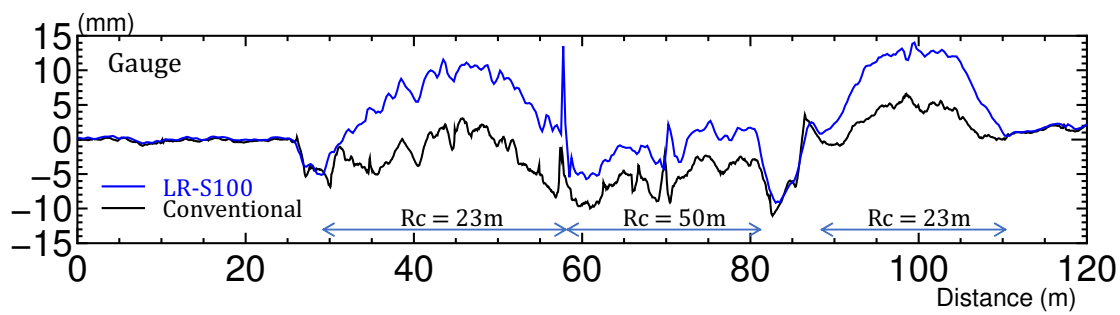

Figure 10: Comparison of gauge irregularity between LR-S100 and conventional measuring trolley along a sharp curve.

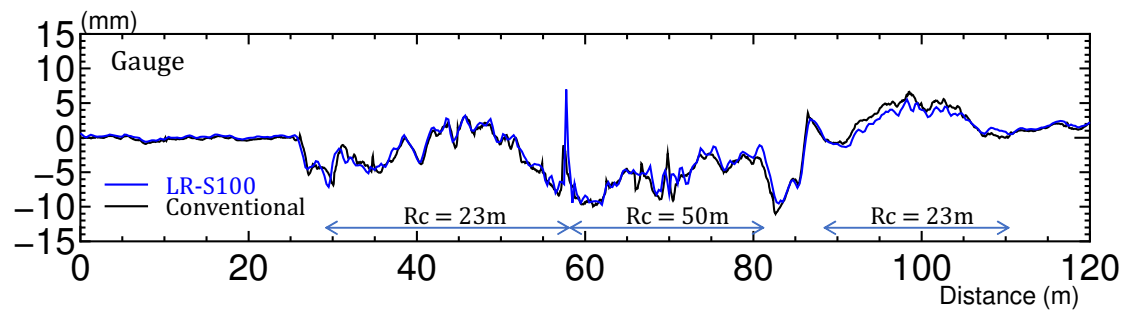

Figure 11: Comparison of compensated LR-S100 gauge and gauge measured with conventional machine along a sharp curve. 


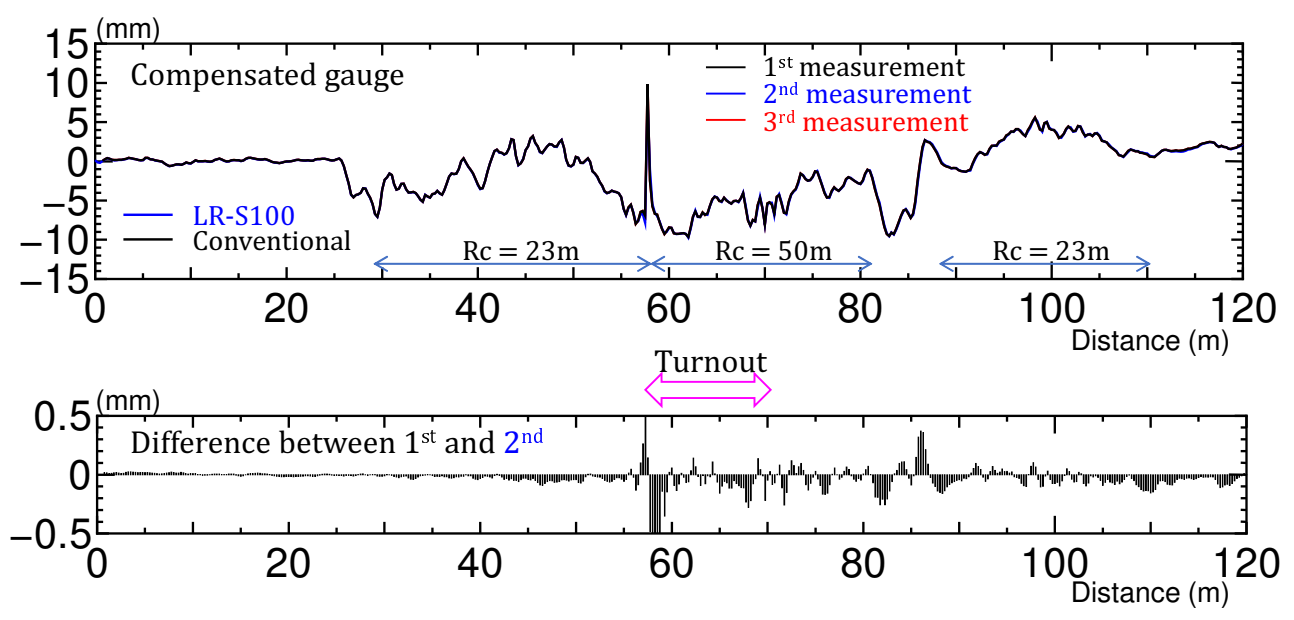

Figure 12: Repeatability of compensated gauge measured by LR-S100.

\section{CONCLUSION AND FUTURE TASKS}

Below is a summary of our conclusion.

(i) We sought to estimate the actual track geometry by applying two reconstruction methods, the inverse filter and Kalman filter, to track irregularity data measured by inertial trolley called LR-S100.

(ii) Because the inverse filter require restoration band restrictions, it is not possible to restore the long wavelength components of the curved section.

(iii) A proprietary method applying the Kalman filter to inverse analysis may make it possible to restore the track geometry of the curved section.

(iv) As an application of the actual track geometry restoration, we proposed a method of compensating for gauge and cant irregularity as measured by a three-wheel inertial trolley. Using the actual track geometry restored by the Kalman filter, we found that it can be compensated for the correct gauge as defined without any additional sensors.

The reconstruction of the actual track geometry is also useful for switch management and other tasks. We will continue to improve restoration precision by using not only inertial trolley but also tamping machine and track recording car data.

\section{ACKNOWLEDGEMENTS}

The authors are grateful to Professor Emeritus A. Yoshimura of Tokyo University of Technology for useful advice on restoring the actual track geometry using a Bayesian estimation filter. The authors would like to thank the development members of DEICY Co., Ltd for their efforts to bring inertial trolley from the laboratory to the commercial product LR-S100.

\section{REFERENCES}

[1] Naganuma, Y., Tanaka, M. \& Ichikawa, K., High-speed track inspection car in the new dr. yellow. Proc. of the World Congress on Railway Research (WCRR 2001), 2001. 
[2] Naganuma, Y., Kobayashi, M. \& Okumura, T., Inertial measurement processing techniques for track condition monitoring on shinkansen commercial trains. Journal of Mechanical Systems for Transportation and Logistics, 3(1), pp. 315-325, 2010.

[3] Yoshimura, A. \& Naganuma, Y., A novel method of reconstructing the railway track geometry using the particle filter and the possibility of the reconstruction of the 3D track geometry. 10th World Congress on Railway Research, WCRR2013, Sydney, Australia, 25-28 November, 2013.

[4] Yoshimura, A. \& Naganuma, Y., Bayesian reconstruction of 3D railway track geometry by particle filter. Proceedings of the Second International Conference on Railway Technology, 2014.

[5] Naganuma, Y. \& Yada, T., Development of truly portable track geometry recording trolley and accompanying new measurement principal. Computers in Railways $X V$ : Railway Engineering Design and Operation, 162(14), pp. 329-342, 2016.

[6] Chen, Q., Niu, X., Zuo, L., Zhang, T., Xiao, F., Liu, Y. \& Liu, J., A railway track geometry measuring trolley system based on aided INS. Sensors, 18(2), p. 538, 2018.

[7] Yoshimura, A., Theory and practice for restoring an original waveform of a railway track irregularity. Quarterly Report of RTRI, 36(2), 1995.

[8] Yoshimura, A., Establishment of theoretical foundation to restoring an original waveform of track irregularity and its application. Railway Technical Research Report, 1336, 1987. (In Japanese.) 